\title{
XII.
}

\section{Beitrag zur Pathologie der Cavernitis- chronica.}

\author{
Von Dr. Ludwig Niehus in Bern.
}

In der Deutschen Chirurgie, und zwar in seinem Buche über „Verletzungen und Krankheiten der männlichen Harnröhre und des Penis", giebt Kaufmann seinem Bedauern darüber Ausdruck, dass wegen Mangel an genaueren pathologisch-anatomischen Untersuchungen die Pathologie der Cavernitis chronica noch als ein sehr unklares Capitel betrachtet werden müsse. Er führt einige von Johnson, van Buren und Keyes unter diesem Titel beschriebene Fälle an, deren eigentliche Natur aber wegen der fehlenden anatomischen Untersuchung nicht feststeht.

Um nun das Wesen der Erkrankungen, welche unter dem Bilde einer Cavernitis chronica verlaufen, zu ergründen, scheint es mir nöthig, dass jeder Fall dieser eigenartigen Affection wenn möglich genau anatomisch untersucht und publicirt werde.

Die vorliegende Arbeit beschäftigt sich mit einem Fall dieser Art.

I. Krankengeschichte.

Anamnese.

Patient Luigi Dotta, 53 Jabre alt, Schreiner von Airolo, stammt von einem gesunden Vater ab, dagegen soll die Mutter mit 36 Jabren an Schwindsucht gestorben sein. Sechs Geschwister leben und sind gesund, eine Schwester starb an einer dem Patienten unbekannten Krankheit. Zwei Söhne des Patienten im Alter von 17 und 19 Jahren sind gesund, eine Tochter starb mit 18 Monaten an Masern oder Scharlach. Die erste Frau des Patienten war gesund, sie wurde im Juli 1879 von einem stürzenden Felsblock erschlagen. Im Juli 1880 verbeirathete sich Patient zum zweiten Mal, seine zweite Frau leidet an einem Uterusfibroid. Im Alter von 40 Jahren machte Patient eine Lungenentzündung durch, sonst war er nie krank. Venerische Erkrankungen werden bestimmt in Abrede gestellt; seit seiner Verheirathung im Jahre 1866 hat Patient nur mit seinen Frauen Ungang gehabt und von denselben seines Wissens sich nie inficirt.

Die ersten Zeichen einer Erkrankung des Gliedes traten schon vor 7 Jahren anf: Anfang des Sommers 1879 wurde Patient von seiner Frau auf Archiv f. pathol. Anat. Bd. 118. Hit. 1. 
etwas Ausfluss aus der Harnröhre aufmerksam gemacht, der Flecken in der Wäsche hinterlassen hatte. Der Ausfuss war wasserbell, steifte die Wäsche nur wenig und wurde von einem später consultirten Arzt für unschuldig erklärt; der Ausfluss hörte in der Folge nie ganz auf. Im November 1879 konnte Patient eines Morgens, nachdem er sich am 'Tage vorher stark angestrengt und erkältet hatte, plötzlich den Urin nicht mehr lassen, bekam Schmerzen in der Blasengegend und bemerkte damals zum ersten Mal eine sehr starke Verengerung der äusseren Harnröhrenöffnung. Sofort auf das Glied und die Unterbauchgegend gemachte Flachssamen-Kataplasmen bewirkten, dass Patient 2 Stunden später wieder leicht und ohne Schmerzen uriniren konnte mit kräftigem Strahl; der Urin war klar. Die Verengerung der Harnröhrenöffnung war verschwunden und häufigerer Harndrang wie gewöhnlich trat nicht ein. Noch zwei Mal wiederbolte sich der Anfall im Winter $1879 / 80$ in gleicher Weise. Nur das dritte Mal im Februar 1880 dauerte es etwas länger, bis Urinentleerung möglich war, auch war der Strahl dünner, weniger kräftig und das Uriniren von brennendem Scbmerz begleitet. Eine Schwellung des Gliedes war zwar nicht zu bemerken, aber die Harnoöhrenöffnung blieb etwas verengert, der Urinstrahl blieb matter, dünner und beim Uriniren waren die näcbsten $3-4$ Wochen hindurch regelmässig etwas brennende Schmerzen vorhanden. Dann gingen auf medicamentöse Behandlung hin sämmtliche Erscheinungen zurück und Patient will bis zum September vollständig gesund gewesen sein, verheirathete sich auch wieder in dieser Zeit.

Ende September 1880, also nacb circa sechsmonatlichem Wohlbefinden, trat wieder plötzliche und absolute Unmöglichkeit ein, den Urin zu lassen. Die wieder angewandten Kataplasmen hatten keinen Erfolg, die Schmerzen in der Blase wurden sebr heftig und dem herbeigerufenen Arzt gelang es nicht, einen ganz feinen Katheter in die Harnröhre einzufübren. Patient wurde in ein warmes Sitzbad gebracht und nach einigen Stunden kam der Urin in ziemlich kräftigem Strahle.

Von jetzt an blieb die Urinentleerung behindert, bald mebr, bald weniger. Die Verengerung der Harnröhre ging nicht mebr ganz zurück, der Urinstrabl blieb matter, kürzer und dünner. Der Arzt gab innerlich Medicinen und machte Einspritzungen mit einer scharfen brennenden Lösung. Gleiche Anfälle wiederholten sich alle parar Wochen; Patient nahm alsdann ein Sitzbad und konnte nach einiger Zeit im Bade uriniren.

In November 1880 bemerkte Patient zum ersten Mal eine harte Schwellung des vorderen Endes der Harnröhre. Diese Schwellung batte die Dicke eines dünnen Bleistiftes, eine Länge von $3-4 \mathrm{~cm}$ und war weder spontan noch auf Berührung schmerzhaft. Sie verschwand in der Folge nicht wieder, wurde aber auch zunächst nicht grösser, sondern blieb sich mehrere Jahre hindurch vollkommen gleich. Die Pebandlung mit kinspritzungen und Bädern wurde den ganzen Winter $1880 / 81$ bindurch fortgesetzt. Im Frühling 1881 erhielt Patient von seinem Arzte eine Reihe kurzer Hartgummisonden, welche er täglich einführen solite; es waren die Nummern 4 bis 12, doch 
brachte er es nie über No. 8 hinaus. Die Anfälle, wie sie oben dargestellt wurden, blieben nun aus; wenn der Urin nicht kommen wollte, brauchte Patient nur eine seiuer Sonden etwa $6 \mathrm{~cm}$ weit einzuführen, und sobald er dieselbe zurückzog, kam der Urin in kräftigem Strahle nach, wäbrend derselbe bei spontaner Entleerung sogleich obne Bogen matt zu Boden fiel. So blieb der Zustand des Patienten mit geringer Besserung oder Verschlimmerung bis October 1884. In diesem Monat trat innerhalb weniger Tage eine sebr starke, etwas harte und sehr schmerzhafte Schwellung des ganzen Gliedes vor dem Scrotum auf. Der Durchmesser soll nach der Beschreibung des Patienten etwa $5 \mathrm{~cm}$ betragen haben. Ein Arzt wurde nicht beigezogen, sondern wieder Flachssamen-Kataplasmen aufgelegt. Die Urinentleerung war trotz der bedeutenden Schwellung spontan möglich, wenn auch mühsam und schmerzhaft. Nach etwa 14 Tagen brach anscheinend die Geschwulst auf, denn es entleerte sich aus der Harnröhre eine ziemliche Menge Eiter. Eine solche Entleerung fand noch 2-3mal innerhalb zweier Tage statt und zwar gleichzeitig mit der Urinentleerung, so dass zuerst Eiter, dann Urin und zum Schluss wieder etwas Eiter abfloss. Die Schwellung des Gliedes soll hierauf vollständig zurückgegangen sein, ebenso die Schmerzen. Die Urinentleerung wurde wieder unbehindert, der Strahl kräftig, so dass er nicht mehr unmittelbar zu Boden fiel. Auch die längst bèstandene Schwellung am vorderen Theil der Harnröhre soll fast völlig verschwunden sein. Patient glaubte sich gänzlich gebeilt und der Winter 1884/85 war einer der besten, den er seit seiner Erkrankung gebabt hatte.

Gegen Frühling 1885 aber wurde der Urinstrabl wieder dünner, matt und gleich zı Boden fallend, die Entleerung mühsam und schmerzhaft, doch war Patient nicbt genöthigt, die Sonde einzuführen, was er vorher immer von Zeit zu Zeit batte thun müssen. Ende Juli und Anfangs August 1885 trat wieder eine allmählich zunebmende harte Anschwellung auf, die in circa 3 Wochen ihre höchste Höhe erreichte. Sie betraf die Glans penis, ferner die Vorhaut, besonder's die linke Hälfte derselben, welche damals mit einem angrenzenden Theil der Haut des Penis eine bedeutende Geschwulst bildete. Diese letztere war hauptsächlich schmerzhaft, sowohl spontan wie auf Druck. Die Urinentleerung war von lebhaftem brennendem Schmerz begleitet, der Strahl matt zu Boden fallend, doch kam der Urin immer noch spontan ohne Nachhülfe der Sonde. Ende August bildete sich ohne Zuthun des Patienten eine kleine Perforation an der unteren Seite des Präputium, etwas links rom Frenulum. Es floss ziemlich viel Eiter ab und die Präputialgeschwulst wurde kleiner. Die Secretion dauerte einige Tage, dann hörte der Eiterausfluss auf, dafür spritzte aber nun bei der Urinentleerung aus der entstandenen Oeffnung jeweilen ein feiner Strabl Uriu. Vorübergebend bat sich im October 1885 die Oeffnung verstopft, so dass kein Urin wehr ausfliessen konnte; es trat daraufhin eine erneute stärkere Anschwellung der betreffenden Präputialbälfte auf. Ende October und Anfangs November öfnete sich die Fistel wieder und gleichzeitig entstanden auf der Eichel in kurzer Zeit eine Menge kleiner Oeffnungen $(10-15)$, aus welchen zuerst etwas kiter ausfloss und 
die in der Folge beim Uriniren den Harn austreten liessen. Zeitweise verstopfte sich ein Theil der Oeffnungen, um sich nach einigen Tagen wieder zu öffnen. Ende August war auch wieder eine harte Anschwellung lüngs der Harnröhre aufgetreten, die sich zuerst nur etwa bis zum Ansatz des Scrotums erstreckte, sich seither aber langsam und allmählich weiter nach hinten fortgesetzt hat. Das Orificium extern, uretbrae hatte sich mit der Schwellung der Glans wieder stark verengt und zugleich begann auch aus der Harnröhre von Zeit zu Zeit tropfenweise etwas Eiter auszufliessen.

Seit dem Herbst 1885 hat Patient auch bäufigen schmerzbaftei Harndrang, so dass er alle 1-2 Stunden, Tag und Nacht, uriniren muss; auch soll der Urin seitdem trübe und die letzten Tropfen gewöhnlich blutig gefärbt sein. Doch glaubt Patient, dass sich im Allgemeinen sein Zustand seit December 1885 eher etwas gebessert habe. Die Geschwulst der Eichel babe wenigstens vorn abgenommen; die Haut derselben sei im Anfang schwarzblau gewesen und habe etwa seit April 1886 eine bessere Färbung bekommen. Aber nach binten bat die Gescbwulst Fortschritte gemacht, so dass die Furche hinter der Glans sich mehr und mehr abgeflacht bat. Ebenso haben die Schmerzen nicht ab-, sondern constant zugenommen, so dass sich Patient bei seiner Aufnahme subjectiv viel schlechter befindet, als früher, trotz der partiellen Abnatme der Geschwulst. Patient will auch seit dem Herbst 1885 bedeutend - etwa um 20 Pfund - abgemagert sein und verspürte viel Müdigkeit in den Beinen.

Am 18. Juni 1886 wurde Patient in die Klinik des Herrn Prof. Dr. Kocber in Bern aufgenommen, dessen Güte ich die Ueberlassung der Krankengeschichte behufs Publication verdanke.

\section{Status.}

Die Untersuchung des Patienten kurze Zeit nach seiner Aufnahme in's Spital ergab Folgendes:

Der allgemeine Ernährungszustand ist noch ziemlich gut, das Fettpolster mässig entwickelt, die Musculatur kräftjg. Die Wangen sind etwas eingefallen, mit ektatischen Gefässen versehen. Die sichtbaren Schleimbäute blass. Die Zähne fehlen zum Theil, theils sind sie schlecht gepflegt und cariös. Die Schleimhäute des Rachens und weichen Gaumens sind leicht geröthet und geschwollen, Geschwüre oder Narben nicht vorhanden. Der Puls zählt 66 Schläge, ist regelmässig, wenig voll und ziemlich leicht zu unterdrücken. Die Respiration 24, regelmässig. Unter der Unterkiefer und zu beiden Seiten des Halses finden sich leicht geschwollene Drüsen. Auch die rechte Orbitaldrüse ist leicht vergrösšert, die linke nicht. In beiden Leistengegenden sind viele erbsen- bis haselnussgrosse Drüsen zu fühlen. Die Füsse sind nicht ödematös.

Ueber der rechten Fossa supraclavicularis und supraspinata ist das Exspirium etwas verlängert, sonst an Lunge und Herz nichts Besonderes zu finden. Leber und $\mathrm{Milz}$ sind nicht vergrössert. In der Unterbaucbgegend einige kleine pustulöse Efflorescenzen. 
Hoden und Nebenhoden beiderseits, ebenso die Samenstränge unverändert. Auch die Scrotalhaut zeigt nichts Abnormes. Der Penis ist von seiner Wurzel am Mons veneris an sebr dick. Sein vorderes Ende, die Glans und die zunächst dabinter gelegene Partie mit der Vorhaut, zeigen die stärkste Schwellung; der grösste Umfang beträgt $17 \mathrm{~cm}$. Die Glans ist allseitig gleichmässig angeschwollen, hat den Durchmesser eines starken Hübnereies - der Corona glandis entsprechend $4 \frac{1}{2} \mathrm{~cm}$ - und ist von derber Consistenz. Ihre Schleimhaut ist bläulichroth, trocken, spröde, der Cutis ähnlich; das Epithel oben hinten, zu beiden Seiten der.Mittellinie vor der Corona, abschilfernd. Ueberall geht die Schleimhaut über die Schwellung hinweg, sie ist in den nnteren zwei [ritttheilen uneben, fachhöckerig, überall von zahlreichen Löchern durchbohrt. Ferner finden sich zu beiden Seiten des Frenulum, mehr rechts als links, erbsengrosse unregelmässige Höcker, an deren Spitze zum Theil ebenfalls kleine Löcher mänden. Der Sulcus glandis ist in den unteren zwei Dritteln statł rinnenförmig eingezogen, flachconvex, die Corona glandis nur leicht angedeutet, geht unmittelbar auf ihn über. In seinem oberen Dritttheil bildet der Sulcus eine flache, wenig vertiefte Rinne. Die Harnröhrenöffnung bildet eine geschlossene Spaite, deren Ründer nur sehr wenig aus einauder gezogen werden können, aber hart und infiltrirt und in Folge leichter Schwellung prominent sind. Druck auf die Glans ist schmerzhaft und bewirkt Ausfluss von dickem Eiter aus dem Orificium uretbrae externum, sowie aus der grossen Anzabl von kleinen Löchern, von welchen die Glans siebartig durchlöchert ist. Auch das Frenulum praeputii ist verdickt und derb. Die Vorhant ist hauptsächlich in ihrer unteren Hälfte, neben dem Freuulum, derb ödematös geschwollen. Diese Schwellung nimmt gegen den Rücken des Gliedes zu allmählich und gleichmässig $a b$, so dass sie in der Mittellinie der oberen Circumferenz nur noch gering ist. Die Vorhant ist hinter die Glans zurückgezogen, lässt den grössten Theil des Sulcus frei. Unten kann sie gar nicht nach vorn gebracht werden, oben Jässt sie sich ein wenig über die Corona glandis hinüberziehen. In der Furche zu beiden Seiten des Frenulum sind ebenfalls Löcher, denn bei Druck auf die Glans entleert sich dort zuerst und am meisten Eiter. Auch in der Vorhaut selber befindet sich in der Mitte einer flachen erbsengrossen Erhebung der Haut eine solche Oeffnung, aus der ebenfalls Eiter ausgedrückt werden kann. Dieselbe befindet sich links neben dem Frenulum am hinteren Ende der Vorhaut, wo letztere in die Haut des Penis übergeht.

Von der Glans aus setzt sich eine harte knollige Verdickung des Corpus cavernosum urethrae nach hinten fort, allmählich abnehmend, um sich am Damm unmerklich zu verlieren.

Der Bulbus uretbrae erscheint weder verdickt noch hart. Die Dicke des Corpus cavernosum urethrae in der Pars pendula ist etwa die eines Zimmermannsbleistiftes.

Die Corpora cavernosa penis sind nicht verändert.

Das Orificium urethrae externum lässt eine Sonde No. I einige Millimeter tief eindringen. Die Katheterisirung ist ganz unmöglich. Die Perfo- 
rationsöffuungen lassen eine gewöhnliche geknöpfte Sonde nur $1-2 \mathrm{~cm}$ tief eindringen, in der Tiefe findet dieselbe derben Widerstand.

Die Urinentleerung ist eine sehr mühsame und schmerzhafte. In Anfang derselben quellen aus einer Unzahl von Perforationen der Glans Urintropfen, welche derselben Aehnlichkeit mit einem Regendouchen-Ansatz verleihen. Später fliesst der Urin mit sehr geringer Kraft aus dem Orificium extern. urethrae aus.

Der Urin ist trïbe, lässt nach einigem Stehen viel grauweissen, fetzigen visciden Bodensatz fallen; seine Reaction ist sauer. Die mikroskopische Untersuchung lässt im Satz viel Eiterzellen und einige Plattenepitbelien, keine rothen Blutkörper erkennen. Die oberen Schichten des Urins entbalten so zu sagen kein Eiweiss, nur ganz minimale Trübungen treten beim Kochen auf.

Die Untersuchung per Rectum ergiebt eine ziemlich starke Vergrösserung der rechten Prostatahälfte, eine geringere der linken, beide sind von derber Consistenz, auf Druck nicht schrerzhaft. Die Samenblasen sind nicht zu füblen. Die Temperatur normal.

\section{Diagnose und Therapie.}

In Rücksicht auf das ungewohnte Bild, welches die Glans und die ibr angrenzenden Partien der Urethra darboten, ein Bild, das durch Annabme anderer Erkrankungen, wie: Carcinom von der Oberfläche der Glans ausgehend, Elephantiasis und Syphilis, keine genügende Erklärung fand; in Anbetracht dessen, dass der Befund einem solchen, wie er durch eine cbronische Entzündung des Schwellkörpers bervorgerufen würde, am meisteu entsprach; gestützt endlich auf die lange Dauer der Affection, die mit zeitweiligen Besserungen und Verschlimmerungen einen Zeitraum von 7 Jahren umfasste, wurde die Diagnose auf Cavernitis chronica gestellt. Auch das Symptomenbild fand durch diese Annahme die leichteste und beste Erklärung. Zwar wurde namentlich wegen der knolligen, von der Glans nacb binten gehenden Verdickungen auch ein von der Schleimbaut der Urethra ausgehendes Carcinom in Frage gezogen, doch hatte diese Erwägung auf die therapeutischen Maassnahmen keinen wesentlicben Einfluss. Vorzugsweise behufs Herstellung eines ungehinderten Urinabflusses, der den Symptomen nach sein Haupthinderniss im Bereiche der Glans hatte, wurde die Abtragung der Eichel bescblossen. Dabei wurde sogleich die Möglichkeit in Aussicht genommen, die Amputation noch weiter nach hinten verlegen $z u$ müssen, falls sich während der Operation die Notbwendigkeit dazu herausstellen würde.

Zum Beginn der Operation wurde daher zur Sicherstellung der Diagnose die Spaltung der Glans vorgenommen. Dabei zeigte sich, dass das Bild von dem eines Carcinoms gänzlich verschieden war; das Aussehen der Urethralschleimhant schloss ferner den Ausgangspunkt von dieser aus. Das an Stelle des Corpus cavernosum der Glans getretene Gewebe schien durch sein gelblich-graues, einem sebr anämischen, etwas speckigen Granulationsgewebe gleichendes Ausseben die Diagnose auf Cavernitis chronica zu rechtfertigen. 
In Folge dieses Befundes wurde die Amputation hinter der Glans mit dem glühenden Paquelin'schen Messer ausgeführt.

Das Präparat wurde dem hiesigen patbologischen Institut überwiesen, dessen Director, Herr Prof. Dr. Langhans mir dasselbe gütigst zur weiteren Untersuchung überliess.

\section{Pathologisch-anatomische Untersuchung. Makroskopischer Befund.}

Die amputirten Theile bestehen aus der Glans, dem Präputium, das zurückgezogen ist und einem angrenzenden Theile des Penis von etwa $1 \mathrm{~cm}$ Länge. Der grösste Durchmesser der Glans beträgt am gehärteten Präparat etwas vor der Corona $4 \mathrm{~cm}$. Ibre Form ist ziemlich normal, abgeseben von einem Verstrichensein des Sulcus. Sie hat eine sehr derbe feste Consistenz. und im Ganzen glatte Oberfäche, die aber mit ziemlich zahlreichen, kleinen, ganz flachen, rundlichen Erbabenheiten versehen ist, in deren Mitte sich kleine Vertiefungen finden. Die Wände der letzteren haben nach Erhärtung in Spiritus eine starke, eigenthümlich braungelbe Farbe, während im übrigen Theil die blasse weissgelbliche Farbe des Spirituspräparates sich findet. Die Erhabenheiten stehen in ziemlich gleichmässig grossen gegenseitigen $\mathrm{Ab}$ ständen von etwa $0,5 \mathrm{~cm}$, hier und da nach vorn bin etwas dichter.

An der Corona glandis, an der Umschlagsstelle des Präputium, findet sich eine Gruppe kleiner, fast kegelförmiger Auswüchse, etwa $3 \mathrm{~mm}$ hoch und an der Basis 2-3 mm dick; diese haben an ihrer Spitze, wie an der Basis kleine grubenförmige Vertiefungen.

Das Gewebe erscheint auf der Schnittfäche graugelb, stark transparent, von eigenthüwlich faserig lappiger Zeichnung. Die Fasern sind weisslich, die kleinen runden, etwas prominenten Lappen graugelb, transparent, die ganze Zeichnung in Farbe und Anordnung sehr der Schnittfläche eines Oterusfibroid ähnlich. Die weisslichen Fasern gehen, seitlich scharf begrenzt, stellenweise in die verdickte Albuginea über. Ferner sieht man zahlreiche schmale Spalten in ziemlich regelmässiger Entfernung, hier und da sich verästelnd, etwas an die Hoblräume des cavernösen Gewebes erinnernd, jedoch durch ungleich breitere Septa getrennt; denn ihre Entfernungen von einander betragen $2-3$, selbst $4 \mathrm{~mm}$. Fast bis an die Operationsfläche setzt sich dieses feste Gewebe fort; blutreiches cavernöses Gewebe ist am frischen Präparat nicht sichtbar.

Von der Schnittfläche lässt sich ein wenig Saft abstreifen, der Jeicht trüb ist und mikroskopisch weisse Blutkörper zeigt, daneben einzelne grosse èpitheliale Elemente, sogar eine Zelle ganz von einer Blase eingenommen, welche letztere 10-15 Lymphkörper enthält.

Die Schleimhaut der Urethra is in unregelmässigen derben Längsleisten emporgehoben, lässt aber sonst keine Veränderungen erkennen.

Das Präparat wurde in Alkohol gebärtet; Stücke davon in Celloidin und in Paraffin eingebettet und mit dem Mikrotom geschnitten. Die mikrosko- 
pische Lntersuchung wurde zunächst an Schnitten vorgenommen, die mit Boraxcarmin und Hämatoxylin gefärbt waren.

\section{Mikroskopischer Befund.}

Während an der normalen Glans die Papillen gegenüber der Epithelschicht, besonders aber gegenüber den interpapillären epithelialen Einsenkungen in den Vordergrund treten, hat sich dieses Verbaltniss an dem vorliegenden Präparate gerade umgekehrt gestaltet: die Lage der geschichteten Epithelien ist bedeutend verdickt, die Papillen entweder sehr niedrig, nur noch durch flache bogenförmige Ausbuchtungen der Epithelschicht angedeutet, oder auch sie sind sehr boch, aber ungemein verschmälert, und in diesen Fällen sind besonders die interpapillären Epithelzapfen von bedeutender Breite. Doch hierbei hat es nicht sein Bewenden, man sieht hin und wieder Gruppen von 2-3-4 solcher interpapillärer Zapfen etwas weiter in die Tiefe gehen, hier mit einander in Verbindung treten und so ein Netzwerk darstellen, in dessen Massen sich gefässfübrendes Bindegewebe findet. Es sind dieses Bilder, die sebr an Schrägschnitte durch dje Epidermis erinnern, bei welehen auch oft mit der Oberfläche zusammenhängende, netzförmig angeordnete Epithelzellstränge sich finden, in deren Maschen die Schrägschnitte von Papillen sichtbar sind und in die auch von unten und den Seiten noch Papillen eindringen.

Oft verbindet sich eine gleiche Anzahl von interpapillären Epitheleinsenkungen zu einem dicken soliden Gebilde, das in einer Breite ron etwa $0,5 \mathrm{~mm}$ bis zu $15 \mathrm{~mm}$ weit in die Tiefe geht und entweder unten stumpf endigt, oder an seinem Ende eine Andeutung beginnender Verzweigung darbietet. In der Mehrzahl der Schnitte findet man auch Epithelzapfen, die bis zu. $1 \mathrm{~mm}$ breit und nicht aus der Vereinigung mehrerer interpapillärer Zellwucherungen hervorgegangen zu sein scheinen, sondern sie sind in ihren oberen zwei Dritteln vollständig compact und aur an ihrem unteren Theil dringen von der Albuginea aus papillenartige Fortsätze in sie ein. Auch diese Zelleinsenkungen erreichen eine Länge von $1-1,5$ иnm. Alle diese tiefer dringenden Zapfen zeigen immer dieselbe Anordnung der Zellen, wie es bei der normalen Epidermis der Fall ist: nach oben, bezw. im Innern der Zellstränge grosse saftreiche, polyedrische Epithelzellen, begrenzt nacb unten, bezw. aussen von einer Lage von cylindriscben Zellen.

In den epithelialen Zelleinsenkungen trifft man ziemlich häufig, in dem übrigen Oberflächenepithel der Glans nur in mässiger Zahl Wanderzellen an, deren Kerne meist ein sehr unregelmässiges verzerrtes Aussehen haben.

An der Albuginea machen sich an verschiedenen Gegenden der Glans verschiedene Verbältnisse geltend. Im Ganzen ist dieselbe in einer mächtigeren Schicht vorhanden wie normal, sie hat eine Dicke von $3-4 \mathrm{~mm}$. Ihre fibrilläre Zeichnung tritt sehr deutlich hervor und die länglichen bläschenförmigen Kerne zwischen den Fibrillen und diesen gleichgerichtet sind in bedeutend grösserer Zahl vorbanden wie in der Norm. Aıch die Gefässe sind ziemlich zahlreich und etwas erweitert, sie zeichnen sich durch eine 
dicke faserige Adventifia aus, die arm ist an länglichen Kernen. Daneben ist eine Infiltration mit einkernigen Lymphkörpern vorhanden, die jedoch eine sebr ungleichmässige ist. Kleine Beerde dicht gelagerter Leukocyten sind in unregelmässiger Weise in der Albuginea vertheilt. Die Meerde sind zum Theil rundlich and gerade in diesen lagern tie Zolien oft so dicht, dass das Bild an einen kleinen Abscess erinnert. Auch in Zügen treten sie auf und diese schliessen sich dann meist an die Gefässe an; da wo ein Gefäss im Querschnitt getroffen ist, umgiebt die Infiltration dasselbe häufig ringförmig.

Am auffallendsten jedoch ist eine Infiltration, welche sich in den oberen Schichten der Albuginea findet und zwar da, wo sich dfe interpapillären Epitbelzapfen etwas weiter in die Tiefe erstrecken. Sie umgiebt die unteren Enden derselben, sitzt diesen fast hutförmig auf und ist uın so stärker, je tiefer die Zellstränge heruntergehen.

Im Vebrigen ist die Albuginea ärmer an Rundzellen, entbebrt dieselben jedoch nirgends vollständig, vielmehr findet sich in allen ihren Schichten eine geringe, aber gleichmässige Infiltration. Immerhin ist der Gegensatz zn dem übrigen Gewebe ein ganz bedentender; gegen das ausserordentlich stark infltrirte und deshalb sebr dunkel gefärbte unterliegende Gewebe der eigentlicben. Glans setzt sich die wenig infiltrirte Albuginea als ganz belles, wie normal erscheinendes Gewebe schon makroskopisch sehr scharf ab.

Die Papillen, in deren Spitze sich nicht selten kleine Gruppen rothbrauner, gelblicher und grünlicher Pigmentkörnchen finden, sind gleichfalls zellreicher wie normal, sowohl an langen Bindegewebs-, wie an Rundzellen; namentlich gilt dieses von solchen, die in der Nachbarschaft von längeren Epithelzapfen liegen, doch ist die Infiltration nur selten stärker, wie in dem grösseren zellenärmeren Theile der Albuginea.

So ist der Befund an der grösseren vorderen Hälfte der Glans. In den hinteren Partien bescbränkt sich die Infiltration nicht auf kleinere Heerde und Züge in verbältnissmässig zellarmem Bindegewebe, sondern hier tritt das letztere eher in den Hintergrund; an Lymphkörpern reichere Partien der Albuginea wiegen vor und grenzen sich nisht scharf von der tiefer sich finden Infiltration ab.

Das Corpus cavernosum der Glans nehmlicb kann man nicht mehr erkennen, von den normaler Weise vorhandenen Hohlräumen ist fast keine Spur mehr zu finden und an Stelle des Schwellkörpers ist ein vollständig compactes Gewebe getreten. Nur an einer Stelle liegen noch 2 Gruppen von kleinen Oeffnungen, die man als Reste des Corpus cavernosum auffassen kann. Die Gruppen sind länglich, haben etwa 1,5 mm im grössten Durcbmesser. Die Oeffnungen, etwa ein Dutzend an Zahl, sind rundlich, zum Theil gebogen und haben bis $7,0,5 \mathrm{~mm}$ Durchmesser. Sie liegen in ziemlich zellreichem Bindegewebe, haben keine besondere Wand, sondern nur ein einfaches Endothel als Auskleidung.

Das zunächst am meisten anffallende an dem Gewebe der Glans ist eine ausserordentlich starke Infiltration mit Lymphkörpern. Die Infiltration betrifft jedoch das vorhandene Gewebe nicht gleichmässig, sondern wird durch zell- 
ärnere und daher hellere Züge faserigen Bindegewebes von geringer Breite in grössere und kleinere Felder von unregelmässiger Gestalt abgetheilt. Im Centrum eines Theiles derselben finden sich grössere und kleinere Mengen von typischem Granulationsgewebe zum Theil mit Riesenzellen; bei anderen, und $Z W a r$ bei der Mehrzahl der grösseren, ist dasselbe ron grossen epithelialen Zellnestern eingenommen, und im Centrum gerade der umfangreichsten treten fast regelmässig grosse klaffende Spalten auf. Diese Heerde erreichen zum Theil eine ganz bedeutende Grösse, sie haben $3-4-6 \mathrm{~mm}$ Durchmesser.

Las Bemerkenswertheste an diesem Gewebe, das die Glans in ibrer ganzen Dicke durchsetzt, sind die erwähnten grossen epithelialen Zellnester. Der Leser wird hier zunächst an Krebs denken; indessen weicht das Bild von dem des gewöhnlichen Carcinoms weit ab. Während bei dem letzteren die Zellnester in der Regel von einer Grösse sind, dass sie erst bei Anwendung einer Vergrösserung deutlich sichtbar werden, können sie hier schon mit blossem Auge leicht erkannt werden. Sie sind von äusserst unregelmässiger, wechselnder Gestalt. Runde Formen sind sehr seiten, länglichrunde siebt man hin und wieder, die meisten sind spindelförmig und sternförmig und stark verzweigt, namentlich die letzteren wiegen vor. Manche sieht man auf einem Schnitt in einer Längenausdehnung von $5 \mathrm{mw}$, während sie eine Breite von $1,5 \mathrm{~mm}$ erreicben. An ibren Enden, oder wo die sternförmigen Nester sich in feine Spitzen ausziehen, schliessen sich ganz schmale Zellstrănge an, die erst bei 10ofacher Vergrösserung sich deutlich verfolgen lassen. Benachbarte Zellnester hängen durch sie zusammen und bilden auf diese Weise ein Netzwerk mit sehr weiten Maschen, denn der Durchmesser derselben beträgt sehr bäufig $3-4 \mathrm{~mm}$. Hie und da sieht man von dem umgebenden Stroma papillenähnliche Fortsätze in diese Nester bereingehen oder auch die Quer- und Schrägschnitte der Papillen in der Peripherie der Nester als kleine runde und längliche Felder ron Bindegewebe.

Die Zellen, aus welchen diese Nester gebildet werden, sind sehr gross, polyedrisch, an den grösseren Feldern häufig im Centrum von länglicher, ja sogar spindelförmiger Gestalt, mit grossem, bläschenförmigem, meist ia Centrum ein grosses Kernkörperchen haltendem Kern. Riffe und Stacheln sind an den meisten sebr deutlich, namentlich an den mehr in die Länge gestreckten Zellen sind sie sehr lang und dem entsprechend auch die Lymphräume zwischen ibnen sehr weit. Nach der Peripherie der Zellnester zu pflegen die Zellen eine mehr eckige Form anzunehmen, dicker und protoplasmareicher zu werden, wie die des Rete Malpighii der Haut, und schliesslich grenzen die Nester durch eine Lage länglicher Zellen, die senkrecht zum Rande des Zejlnestes gestellt sind, dieses gegen das angrenzende Gewebe ab. Diese letzteren Zellen haben oft deutlich Cylinderform, dunkles körniges Protoplasma und etwas kleinere dunklere, aber noch deutlich bläschenförmige Kerne, sind bänfig aber auch spindelförmig and senien dann ihr peripherisches Ende in das angrenzende Gewebe ein. Vielfach wird jedoch die äusserste Peripherie der Epithellzèllnester von abgeplatteten Zellen gebildet und gerade die äusser- 
sten, welche die Grenzschicht bilden, sind am stärksten abgeplattet und der Grenzlinie zwischen Epithel und Stroma parallel angeordnet.

Dieses Verhältniss sieht man sebr schön an den Zellnestern von rundlicher Form. Bei diesen sind die inneren Zellen gross, rundlich eckig, Protoplasma und Kern hell; nach der Peripherie bin werden sie dann schmaler und dunkler, nebmen Halbmond- und weiter Sichelform an und liegen schliesslich wie Zwiebelschalen an einander.

Auch da, wo sich die Felder von Epithelzellen verschmälern, ziemlich spitze Ecken bilden oder Ausläufer aussenden, verändern die Zellen in auffälliger Weise ihr Aussehen. Nach diesen Ecken und Ausläufern hin werden sie allmählich kleiner, ihr Protoplasma dunkler; der Zellleib verschmälert sich, der Kern wird gleichfalls länger, dunkler und dünner und der Ausläufer gleicbt fast einem Strang von länglichen Bindegewebszellen, der mit einem anderen von einem benachbarten Zellnest abstammenden zusammenstösst oder nach längerem oder kürzerem Verlauf obne vollständig scharfe Grenze in dem übrigen Gewebe sich verliert.

Bemerkenswerth ist noch eine ziemlich gleichmässige Vertheilung von meist mebrkernigen Leukocyten in der Mehrzabl der epithelialen Zellnester. Dieselben sind oft so zahlreich vorbanden, dass auf den ersten Blick die grossen blassen Kerne der Epithelzellen übersehen werden können oder doch sehr in den Fintergrund treten. Die meisten dieser Leulsocyten liegen in den intercellulären Saftläcken und nehmen vielfach, der Form und Enge der Passage sich anpassend, eine sehr lange, schmale oder auch verzweigte Gestalt an. Wie im frisch abgestreiften Saft, finden sich auch im Schnitt sebr bäufig in den Epithelzellnestern Zellen, die eine kleinere oder grössere Vacuole mit einem oder mehreien Eiterkörperchen als Inhalt besitzen.

Die Epithelzellnester sind zum grössten Theil nicht vollständig solid, sondern haben ìm Innern Höhlen, die manchmal leer sind und dann aussehen, als wenn ein Theil der Zellen ausgefallen wäre. Diese Höhlen, welche identisch sind mit den schon makroskopisch sichtbaren und am frischen Präparat beschriebenen Spalten, ahmen im Ganzen die Gestalt der Zellennester nach; sie sind rundlich bei runden Nestern, länglich wenn die Nester in die Länge gestreckt sind, ja bei sternförmigen oder verzweigten folgen sie zum Theil deren Verzweigungen. Sie bilden bei vielen den grösseren Theil der Zellnester und baben dann nur noch eine schmale Schicht von Epithelzellen als Begrenzung, ja an beschränkten Stellen ist auch diese noch unterbrochen und das zellreiche Stroma, in dem die Zellnester sich finden, bildet hier den Rand der Höhle. Bei manchen ist die Höhle nur noch an ganz kleinen Randpartien von Resten von Epithellagen begrenzt, derr grösste Umfang wird von kleinzellig infiltrirtem Gewebe gebildet.

Die grossen Höhlen haben somit auch zum Theil annähernd Dimensionen wie die Epithelzellnester; man findet solche von $5 \mathrm{~mm}$ im grössten Durchmesser und manchmal bis $1 \mathrm{~mm}$ Breite. Kleinere Höhlen sind in der Regel noch von einer mächtigeren Schicht von Epithelzellen umgeben. Viele Höhlen sind nicht leer, sondern zeigen eine ganze oder theilweise Ausfüllung mit 
dicbten Masten von mehrkernigen Leukocyten, zwischen denen sich auch noch spärliche Epithelzellen finden können.

Wenn schon die noch in der Albuginea endigenden verlängerten interpapilären Epithelzapfen an ihren unteren Ende von einer Zone dichter Infiltration mit Leukocyten umgeben sind, so ist dieses bei den beschriebenen epithelialen Zellnestern noch in viel höberem Maasse der Fall. An diese grenzt zunächst ein Gewebe, das so dicht mit Lyenphkörpern infiltrirt ist, dass man eine weitere Structur an demselben nicht mehr erkennen kann. Fs sind auch hier einkernige Leukocyten, nur selten findet man mehrkernige zwischen denselben. In wechselnder Anzahl, bald häufig bald selten an verschiedenen Stellen der gleichen Felder und noch wehr bei Vergleichung verschiedener Felder, finden sich in diesen Infiltrationsheerden Gefässe, die das Lumen einer Capillare, auch etwas mebr oder etwas weniger haben, aber mit dicker fasriger Adventitia versehen sind. Mit der Entfernung von dem Epithelzellnest nimmt die Dichtigkeit der Infiltration etwas $a b$, eine fasrige Grundsubstanz wird stellenweise deutlich und schliesslich tritt ein verbältnissmässig zellarmes Bindegewebe hervor, das nur wenig infiltrirt ist, und scheidet das ganze Feld yon einem benachbarten Heerde. Indessen ist damit die Infiltration des Stronas mit Lymphkörpern noch nicht abgeschlossen, sie erstreckt sich auch noch auf Stellen, wo keine Epithelzellnester sich finden. Ich komme darauf zurück.

Im Centrum anderer, aber nur einer Minderzahl der Infiltrationsheerde findet sich statt der Epithelzellnester ein Gewebe, das ich schon weiter oben mit dem Namen Granulationsgewebe bezeichnet habe; denn es erinnert vielfach durch seine Zellformen und deren Anordnung an die infectiösen Granulationsgeschwülste. Dasselbe tritt durch sein blasseres Aussehen als helleres Centrum in einem Infiltrationsheerde hervor, ist von rundlicher Gestalt und hat bis $2110,5 \mathrm{~mm}$ Durchmesser. Es besteht in der Hauptsache aus epithelioiden Zellen, deren bläschenförmige Kerne gut sichłbar sind, während die Zellgrenzen neben dem sehr blassen Protoplasma nicht hervortreten; auch werden dieselben oft durch eine ziemlich starke Infiltration mit meist mehrkernigen Lenkocyten verdeckt. Die Kerne sind nicht von gleicher Grösse, die meisten nur sebr wenig grösser, als einfache Kerne weisser Blutkörper, andere grösser, bis doppelt so gross, und dann gewöhnlich von ovaler Form; die Rernsubstanz ist blass. Zwischen diesen Zellen, im Allgemeinen mehr in den mittleren Partien eines solchen rundlichen Heerdes, aber nicht gerade immer im Centrum, finden sich häufig Riesenzellen, oft in mehreren Exemplaren, die ausserordentlich denen gleichen, welche if Tuberkeln vorzukommen pflegen. Sie baben ein belles feinkörniges Protoplasma and zahlreiche grosse ovale bläschenförmige Kerne, die wandständig und radiär angeordnet oder an einem Pol der Zelle angehäuft sind. Sie sind in einigen Schnitten um eine rundliche Höble angeordnet, die sich im Centrum eines solchen rundlichen Granulationsheerdes findet und einen Durchmesser von etwa $0,1 \mathrm{~mm}$ hat. Der Rand der Höble ist unregelmässig gestaltet, von epithelioiden Zellen gebildet, die zum Theil in dieselbe 
vorspringen. Die Riesenzellen treten ziemlich dicht an diesen Rand heran. Zwischen den epithelioiden Zellen findet sich eine ziemlich starke Infiltration mit mebrkernigen Lieukocyten, die nach der Peripherie bin allmählich durch eine solche von einkernigen ersetzt wird. In der letzteren sind, wie dieses in der Nachbarschaft der Epitbelzellnester ähnlich der Fall ist, zablreiche Gefüsslumina sichtbar, wie jene, meist etwas weiter als Capillaren, wit dicker faseriger Wand versehen.

Bei anderen ähnlichen Heerden fehlt die Höhle im Centrum, auch sind die Riesenzellen nicht immer in mebreren Exemplaren, bei den grösseren jedoch häufig vorhanden, während sie bei den kleineren meist fehlen. Es kommen aber auch Heerde vor aus epitbelioiden Zellen und Riesenzellen gebildet, wo keine Infiltration mit mebrkernigen, sondern nur eine solche mit einkernigen Leukocyten vorhanden ist, und dans finden sich auch schon im Centrum in der. Nähe der Riesenzellen kleinkalibrige Gefässe mit dicker faseriger Wand. Bei denen, die keine centrale Höhle und beine Riesenzellen enthalten, ist die Infiltration mit mehrkernigen Leukocyten sebr gering; nur mässig zahlreiche einkernige finden sich, und mit scharfer Grenze setzt sich meist dieses hellere Gewebe gegen die umgebende starke Infiltration ab.

Die Felder kleinzelliger Infiltration obne Epithelzellnester und ohne Granulationsgewebe im Innern treten an Zahl und Grösse gegenüber den beschriebenen in den Hintergrund. Dieselben werden von dicht gelagerten einkernigen Leukocyten gebildet, zwischen denen sich sehr selten ein mehrkerniges findet. Sie sind in Form von unregelmässigen Feldern und Zügen neben und 2 wischen den grösseren vorhanden und enthalten nur selten ein mittelgrosses dickwandiges Gefäss im Inneren. Zwar kommen auch in seltenelı Fällen in ihnen kleine spaltförmige Höhlen obne Inbalt und von ziemlich unregeluässiger Begrenzung vor, doch zeigt das Gewebe, welches die Ränder bildet, dieselbe gleichmässige Infiltration mit einkernigen Leukocyten, wie an der Peripherie des Heerdes; es treten keine mebrkernigen Lymphkörper in nennenswerther Zahl auf, so dass nicht eiterige Einschmelzung des Gewebes, sondern Artefacte vorzuliegen scheinen.

Die einzelnen, so verschiedenartigen Heerde kleinzelliger Infiltration sind llurch Züge faserigen Gewebes, untermischt wit Bündeln glatter Muskelfasern, von einander getrennt, das im Verhältniss zu jenen kernarm und hell erscheint. Doch ist dasselbe im Vergleich mit dem normalen Gewebe der Glans ebenfalls von bemerkenswerthem Kernreichthum. Die langen bläschenförmigen Kerne zwischen den Bindegewebsfibrillen sind vermehrt und auch einkernige Leukocyten sind in ziemlich gleichmässigen Abständen in mässiger Zahl vorhanden. Stellenweise ordnen sich diese in langen Reihen an, von denen bisweilen 2-3 neben einander liegen, und zwar namentlich $z$ wischen Zügen glatter Muskelfasern, wenn solche noch zwischen oder an Stelle des Bindegewebes auftauchen und zur Abgrenzung der einzelnen Infiltrationsheerde von einander beitragen. Die Fasern der Bindegewebszüge liegen alıch, abgesehen von den durch die Infiltration aus einander gedrängten, oft nicht unmittelbar au einander, sondern baben spaltförmige oder rund- 
liche Lücken zwischen sich, in denen etwa 2-4 Lymphlörper Platz fänden. Sie enthalten aber of keine oder nur ein solches und sind im Uebrigen Jeer, haben auch wohl nur etwas lockere feinkörnige Masse als Inhalt. Sie besitzen keine Auskleidung von Endotbel, sind also als mechanisch durch den Urin hervorgebracht anzuseben, del sich seinen Weg durch das Gewebe der Glans nach der Oberfläche hin gebahnt hat.

Wir haben also als hauptsächlichste und interessanteste Veränderung gefunden: grosse Infiltrationsheerde mit umfangreichen Epithelzellnestern im Innern, welche meist schon makroskopisch gut sichtbar sind und in Form von Zügen das Gewebe der Glans durchziehen. Ihre bedeutende Grösse, ihre scharfe Begrenzung, der fast vollständige Mangel der cavernösen Räume legen die Annahme nahe, dass es sich um Ausfüllung der Blutrïume des Sehwellkörpers handle, und führen weiter zu der Erwägung, ob nicht eine Wucherung der Endothelien in den letzteren stattgefunden, die Zellnester also einen endothelialen Urspruug haben. Doch genauere Betrachtung der Zellnester thut dar, dass die Form derselben sehr selten derjenigen entspricht, welche man bei Ausfüllung von cavernösen Bluträumen erwarten müsste, nur ganz vereinzelt finden sich runde oder länglich runde, von gebogenen gar nicht zu reden; die grösste Mehrzahl hat vielmehr eine so unregelmässige Gestaltung, dass der Versuch unmöglich ist, dieselben auch nur annähernd dem System von cavernösen Bluträumen anpassen zu wollen. Besonders die langen schmalen Ausläufer, welche von den meisten Zellnestern ausgehen, sind mit dieser Annahme nicht gut in Einklang zu bringen. Auch wäre wohl anzunehmen, dass das Wachsthum ein rascheres gewesen sein würde, wenn die Ausbreitung auf solchem überaus günstigem, durch das Hohlsystem präformirtem Wege statgefunden hätte.

Für eine Abstammung von den Endothelien der cavernösen Bluträume ist noch weniger ein Anhaltspunkt vorhanden. Form und Anordnung der Zellen werden sofort auch den leisesten Zweifel beseitigen, da diese ihre Natur als Abkömmlinge geschichteter Epithelien klar stellt.

Es kann also nur noch betreffs ihres Ursprungs die Frage aufgeworfen werden: Stammen sie von den geschichteten Epithelien des in der Glans verlaufenden Theils der Urethra oder stammen sie von denen der Oberfläche der Glans ab? 
Ich muss von vornherein bemerken, dass hier in meinen Beobachtungen eine Lücke sich befindet; es betrifft dieses das Verhältniss der epithelialen Zellnester zum Epithel der Urethra. Manches weist daranf hin, dass sie mit dem letzteren in $\mathrm{Za}$ sammenhang gestanden haben können. Während des Lebens wurde, wie sicher constatirt ist, aus den kleinen Oeffnungen der Glans das Abfliessen des Harnes bemerkt. Der Harn floss also offenbar durch die Spalten, welche sich in den epithelialen Zellnestern befinden, hindurch, und es können die letzteren mit dem Lumen der Urethra communicirt haben; ebenso kann an den Communicationsstellen auch das Epithel der Urethra mit den epithelialen Zellnestern in Zusammenhang gewesen sein. Leider wurde aber die Schleimhaut der Urethra bei der ersten mikroskopischen Untersuchung aus Likenntniss der eben erwäbnten Verhältnisse nicht genügend beachtet. Indessen ist hervorzuheben, dass direct bei der Operation die Urethra gespalten wurde. Man fand die Schleimhaut in derben unregelmässigen Längsleisten emporgehoben, grössere Oefinungen aber wurden an derselben nicht bemerkt. Vielleicht, dass auch diese Communicationen nicht an dem Theil der Urethra innerhalb der Glans, sondern weiter hinten zu suchen waren, es können hierüber nur Vermuthungen aufgestellt werden.

Dagegen lässt sich mit aller Sicherheit nachweisen, dass die erwähnten epithelialen Zellnester mit dem Oberflächenepithel der Glans zusammenhängen und zwar an den Stellen, die schon makroskopisch als kleine rundliche flache Erhabenheiten an der Glans auftraten. Hier senken sich grosse Gruppen von 8--12 interpapillären Epithelzapfen von wechselnder Breite tiefer in das Gewebe der Albuginea ein und treten schon in dieser durch breite seitliche Aeste unter einander in Verbindung. Sie bilden so längliche und runde Maschen, die durch kernreiches Bindegewebe ausgefüllt werden und an Quer- und Schrägschnitte durch Papillen erinnern. $V_{\text {on }}$ den oben beschriebenen kürzeren Zapfen von ähnlichem Aussehen, die noch in der Albuginea endigen, unterscheiden sie sich ausser durch, jhre grössere Länge noch durch ihr seltenes Vorkommen und schliessen dadurch auch einen gegenseitigen Zusammenhang aus. Denn während von jenen in jedem Schnitt mehrere zu finden sind mit der stetigen Infiltra- 
tionszone am die unteren Enden, treten diese, entsprechend den weiten Abständen der rundlichen Erhabenheiten an der Oberflüche der Glans nicht in allen Schnitten hervor. Sodann fehlt bei den kürzeren in allen Fällen eine Höhlenbildung, während bei den längeren vielfach im Innern eine kanalartige Höhle auftritt, die entweder leer oder von mehrkernigen Leukocyten ausgefüllt ist.

Diese, vom Oberflächenepithel ausgehenden Zellzapfen, welche eine Breite von $1 \mathrm{~mm}$ erreichen, durchsetzen die Albuginea, deren Gewebe in der Nähe derselben eine stärkere Infiltration mit einkernigen Lymphkörpern aufweist, dringen in das tiefere Gewebe vor und gehen hier in die beschriebenen grossen Zellnester über. Bei vielen ist die anstossende Albuginea in ihrem grösseren oberen Theil nicht stärker infiltrirt, als dieses in weiterer Entfernung von dem Zapfen der Fall ist, und erst in den tiefsten Schichten derselben oder beim Eintritt in das eigentliche Gewebe der Glans tritt stärkere Infiltration auf.

Einige dieser Stränge begiunen schon in den tieferen Schichten der Albuginea sich zu grossen Zellnestern auszubreiten, deren grösster Durchmesser meist senkrecht zu der Richtung des Stranges liegt. Und gerade in diese, die auch, wie die tiefer gelegenen, mit einer dichten, aber schmäleren Infiltrationszone umgeben sind, kann man sehr zahlreiche Papillen-ähnliche Fortsätze des angrenzenden Gewebes von oben, von unten und von den Seiten her eindringen sehen; namentlich von unten her ist dieses sehr häufig der Fall. Auch centrale Höhlenbildungen werden bei diesen oberflächlicher gelegenen vielfach beobachtet, und zwar Höhlen, die an Grösse gegen die tiefer gelegenen nicht zurückstehen.

Die Höhlen innerhalb der Zellnester setzen sich in die der zugehörigen Zellstränge fort, die grösseren, indem sie sich nach der Oberfläche hin verjüngen; sie münden schliesslich in der Mitte der flachen Erhabenheiten auf der Glans aus, entsprechend den Gruben, welche schon makroskopiseh an diesen auffielen und aus denen sich vor der Operation Eiter und Urin entleerte.

Wenn man eine der kegelförmigen Erhabenheiten, die sich in einer kleinen Gruppe an der Umschlagsstelle des Praeputium hefinden, auf der Schnittfäche betrachtet, so hat sie das Aus- 
sehen eines colossalen papillenartigen Fortsatzes, den das Gewebe der Glans nach oben hin aussendet. Er besteht der Hauptsache nach aus Bindegewebe, das continuirlich von dem tiefer liegenden Gewebe in den Kegel übergeht.

Eine Abgrenzung der Albuginea, wie an den vorderen Partien der Glans, ist hier hinten nicht möglich; einige Bindegewebsbündel scheinen schon unterhalb' des Niveaus derselben aufzutauchen und lassen sich bis in die höchsten Partien des Kegels verfolgen, andere verflechten sich in der mannichfaltigsten Weise. Diese Bindegewebszüge sind ärmer an langen bläschenförmigen Kernen wie die Albuginea der Glans an den Stellen, wo sie hervortritt, aber ziemlich reich an einkernigen Leukocyten, die in Reihen und Gruppen auftreten.

Auf die Oberfläche des Kegels schlägt sich die Epithelbekleidung der Glans an dessen Basis um und überzieht denselben vollständig. Diese epitheliale Bekleidung des Kegels zeigt ein den übrigen Theilen der Glans analoges Verhalten: Verdickung der Epithellage im Allgemeinen, Verlängerung von interpapillären Zapfen und stärkere Infiltration des Gewebes an deren unteren Enden. Da, wo sich makroskopisch Oeffnungen an den Erhabenheiten zeigten, an der Spitze wie an der Basis, nehmen Epithelzapfen ihren Ursprung, die bis zu $1 \mathrm{~mm}$ Breite haben. Sie sind mit einem centralen Kanal versehen, dringen in das tiefere Gewebe der Glans ein und verhalten sich dort, wie die oben beschriebenen.

Um festzustellen, ob die Erkrankung der Glans auf die. Invasion, bezw. Gegenwart von pflanzlichen Mikroorganismen zurückzuführen sei, wurden Schnitte des Tumors nach den verschiedensten Methoden gefärbt, die eine Tingirung der eventuell vorhandenen Bakterien versprachen, doch wurde diese Arbeit nicht mit Erfolg gekrönt. Die Anwendung von Gentianaviolett, Methylenblau und Fuchsin, die Gram'sche, die Gram-Weigert'sche Methode und die Färbung auf Tuberkelbacillen waren vergeblich, keine derselben hatte ein günstiges Resultat.

Doch traten bei dieser Gelegenheit in dem Tumor andere Gebilde hervor, deren Beschreibung wegen ihrer besonderen Eigenthümlichkeit einiges Interesse beanspruchen könnte. 


\section{8}

Zunächst fallen bei der Färbung mit Gentianaviolett ziemlich zahlreiche Mastzellen (nach Ehrlich) auf. Dor Kern ist nur an wenigen als heller Fleck zu sehen, die meisten scheinen solide Häufchen von stark gefärbten feinen Körnern zu bilden. Sie finden sich überall im Bindegewebe, sowohl der Albuginea wie der tieferen Schichten, um so zablreicher, je kernreicher das Gewebe, am häufigsten in der Nähe der kleinzelligen Infiltrationsbeerde; selten liegen sie innerhalb solcher Heerde und höchstens in den peripherischen Partien derselben sind sie noch sichtbar. In den epithelialen und epitheloiden Zellgruppen fehlen sie vollständig.

Ein ähnliches Verbalten, wie diese Mastzellen, sowohl was die Zahl anbetrifft wie auch in topographischer Beziehung, zeigt ein anderer Bestandtheil des Tumors, der sich durch seine Seltsamkeit besonders auszeichnet.

Fast überall nehmlich, wo noch faserige Partien mit mässiger Infiltration in. der Glans vorhanden sind, werden durch Färbung mit Gentianaviolett kleine runde Körpereben tingirt, die fast sämmtlich bei Anf- und Niederschrauben des Tubus Kugelform zeigen, nur selten haben sie einen kleinen flachen Vorsprung. Sie finden sich nur sehr selten in einzeln liegenden Exemplaren, etwas häufiger zu zweien und dreien, in der Mebzabl jedoch in Gruppen von 6-10 Individuen. Oft liegen sie noch zahlreicher bei einander und Gruppen von 25-30 sind keine Seltenheit. Die einzeln liegenden baben meist die bedeutendste Grösse, sie können weisse Blutkörper übertreffen und baben nicht selten $0,012 \mathrm{~mm}$ Durchmesser. Wenn die Gruppen klein sind, übertrifft eine oder zwei die Genossen oft um ein Bedentendes, die grösseren baben dann einen Durchmesser von 0,006-0,012 mm, während die übrigen nut $0,002-4 \mathrm{~mm}$ messen. Ist die Anzahl eines Häufchens grösser, so sind die Kugeln in der Regel klein und annähernd gleich gross, und zwar gewöhnlich um so kleiner, je zahlreicher sie sind. Da, wo etwa 20-30 zusammen liegen, bleiben sie meist in den Grenzen von $0,001-0,003-4 \mathrm{~mm}$ Durchmesser.

Die Gruppen sind von unregelmässiger Form, oft rundlich, und liegen in Spalten und Lücken des Gewebes, die durch Auseinanderweichen seiner Fasern bedingt sind. Da auch meist in ibrer nächsten Nachbarschaft Infiltration mit Lymphkörpern vorbanden ist, so sehieben sich auch wohl noch Leiber von solchen Zellen zwischen die einzelnen Kugeln etwas ein, doch ist nirgends eine Andeutung vorbanden, dass in Bezug auf die Genese eine Beziehung der Lympbkörper zu den Kugeln bestände. Vielmebr haben die Kugeln, ausnahmslos ein vollständig homogenes Aussehen, sie sind ganz gleichmässig gefärbt und keinerlei Kerne oder Körner sind in denselben zu entdecken. Wenn sie nicht zu stark gefärbt sind, bleibt an der Peripherie ein homogener, glasheller, glänzender Saum ungefärbt, der bei den Kugeln mittlerer Grösse, 0,0045-0,007 Durehmesser, am deutlichsten und breitesten erscheint, er erreicht bei diesen eine Breite von $0,001 \mathrm{~mm}$ und ist gegen das gefärbte Innere der Kugel ziemlich scharf abgesetzt. Bei den kleineren Kugeln ist dieser Ring meist weniger deutlich, bei den grösseren in der Regel nur sehr schmal oder gänzlich unsichtbar, ja sogar das Umgekebrte kommt 
vor, dass sich nehmlich die Peripherie stärker färbt, während das Centrum heller bleibt.

Wie mit Gentiavaviolett, so werden die Kugeln aucb nach der Gram'schen Methode zum Färben der Bakterien gut gefärbt, noch intensiver bei Anwendung der durch Weigert angegebenen Modification der Gram'schen Färbung (Entfärbung mit Anilin). In Schnitten, die mit Boraxcarmin (Entfärbung mit verdünnter Salzsäure) oder Hämatoxylin gefärbt sind, treten sie nicht deutlich bervor, da sie diese Farbstoffe nicbt annebmen. Sie fallen in solchen Schnitten nur durch einen gewissen Glanz auf, namentlich wenn man in nicht zu stark Jichtbrechenden Medien untersucht, und erinnern dadarch sehr an Amyloid. Sie sind vollständig homogen wie dieses, doch leicht durch Anwendung von Jodlösung von solchem zu unterscheiden, denn sie nehmen nicbt, wie das Amyloid, einen dunkelbraunen Farbenton an, sondern werden, wie das übrige Gewebe, nur leicht gelb gefärbt.

Im 24. Band des Archivs für experimentelle Pathologie und Pharmakologie S. 122 und 123 beschreibt Sachs eigenthümliche runde Gebilde, die mit der von Heidenhain angegebenen Haematoxylin - Kali - bichromicum-Färbung tiefschwarz gefärbt wurden. Sachs fand diese vereinzelt im Bindegewebe normaler Mägen, in grosser Anzahl in solchẹn, die pathologische Veränderungen zeigten, und zwar namentlich in den oberen Schichten der Schleimhaut, in Spalten und Lücken des Gewebes. Er beschreibt sie wie folgt: "Sie sind gewöhnlich kugelrund, doch sitzen ihnen gern kleine Buckel auf. Ihr Volumen wechselt, gewöhnlich entspricht es dem einer mittelgrossen Belegzelle, doch sind sie manches Mal viel grösser, dann wieder viel kleiner und rothen Blutkörpern an Grösse vergleichbar. Oft liegen zahlreiche kleine Schollen so zusammen gruppirt, dass das Ganze wie eine dicht behangene Traube aussieht. Ungefärbt erscheinen sie mattglänzend, aus völlig homogener Substanz bestehend, in deren Innerem oft kleine Vacuolen, Kanälchen und Spalten zu erkennen sind, ferner Kerne und Zellfragmente, die wahrscheinlich von eingewanderten Leukocyten herrühren."

Da ich nach der Beschreibung von Sachs vermuthen musste, ähnliche oder gleiche Gebilde vor mir zu haben, so färbte ich auch nach der Methode von Heidenhain und erhielt dabei die gleiche tiefschwarze Färbung der kugligen Gebilde, wie Sachs. Weniger Uebereinstimmung mit den Färbungsresultaten von Sachs zeigten diejenigen, welche ich bei Prüfung der Kugeln auf Hyalin nach v. Recklinghausen erhielt. Während Sachs 
seine Schollen mit Fuchsin, Eosin, Carmin und HaematoxylinAlaun gut färben konnte, gelang mir dieses nur mit Fuchsin. Bei Anwendung von Alauncarmin und Eosin blieben die Kugeln ungefärbt, ebenso bei Boraxcarmin und Haematoxylinalaun, was ich schon früher erwähnt habe.

\section{Wesen der Erkrankung.}

Wenn auch das makroskopische Aussehen der Glans sowohl von aussen wie auf der Schnittfläche in nichts an das Vorhandensein epithelialer Zellwucherungen erinnerte, so stellt doch die mikroskopische Untersuchung den Charakter des Tumors im Wesentlichen als den einer epithelialen Neubildung ausser Frage, und zwar als einer solchen, die ihren Ausgangspunkt von den geschichteten Oberflächenepithelien der Glans nimmt. Man könnte allerdings annehmen, dass die Tyson'schen Drüsen den Ausgangspunkt bildeten; es wären dann die in der Albuginea verlaufenden, verhältnissmässig schmalen Zellstränge als die veränderten Ausführungsgänge anzusehen, welche die durch bedeutendere Wucherung der Drüsenepithelien entstandenen grossen tieferen Zellnester mit dem Oberflächenepithel verbänden. Es ist mir zwar an den normalen und pathologischen Glandes, die ich auf das Vorhandensein von Talgdrüsen untersuchte, nicht möglich gewesen, deren nachzuweisen, die eine ähnliche Verbreitung darböten, wie die Zellstränge und Zellnester. Doch sagt Kölliker in seinem "Handbuch der Gewebelehre" S. 148, dass die Tyson'schen Drüsen sehr unbeständig seien und bald in höchst geringer Anzahl (2-10), bald in grosser Menge, selbst zu hunderten sich fänden. Demnach könnten diese als Ausgangspunkt wohl in Betracht gezogen werden.

Die Veränderungen, welche sich an dem Epithelüberzuge neben diesen tiefgehenden Zellsträngen finden, weisen jedoch mehr darauf hin, dass wir es mit einer vom Deckepithel der Glans ausgehenden epithelialen Infiltration zu thun haben. Eine Verdickung der Epithelschicht gegen die Norm ist auf der ganzen Glans vorhanden und bald mehr bald weniger tief in die Albuginea eindringende Gruppen von interpapillären Zapfen, die in ziemlich reichlicher Zahl sich finden, stellen die Uebergänge dar zu denen, welche die Albuginea durchbrechen und sich in dem 
tieferen Gewebe zu umfangreichen Zellnestern ausbreiten. Die Albuginea besteht aus einem festen derben Gewebe, dessen geringer Reichthum an Gefässen nur eine mässige Ernährung bedingt, die ihrerseits nicht den günstigsten Einfluss auf die Ausbreitung der Zellstränge innerhalb derselben haben konnte. Solcher Zellstränge, welche sich noch innerhalb der Albuginea zu grösseren Nestern gestalten, sind deshalb nur wenige vorhanden. Erst wenn sie den Widerstand der derben Albuginea überwunden haben und in das besser ernährte eigentliche Gewebe der Glans vorgedrungen sind, entfalten sie ein iippigeres Wachsthum und erhalten sie eine grössere Ausdehnung. Meine Zweifel an der Annahme, dass sie dabei die cavernösen Bluträume benutzt haben könnten, habe ich schon weiter oben begründet.

Neben dem Hauptmomente, das den vornehmsten Charakter des Tumors klar legt, nehmlich dem Zusammenhang der Zellnester mit dem Oberflächenepithel, sind auch die übrigen Erfordernisse vorhanden, welche die Annahme eines Epitheliums stützen: die Form und Anordnung der Zellen. Denn die grossen polymorphen Zellen mit bläschenförmigen Kernen und lang gezähnten Rändern, die mehr cylindrischen Zellen, welche den Saum der Zellhaufen bilden, vervollständigen den Beweis.

Schon oben habe ich hervorgehoben, dass dabei nicht an ein gewöhnliches Cancroid gedacht werden darf; mit einem solchen hat die epitheliale Infiltration nichts gemein in ihrer Vertheilung, wenn auch die Abstammung der Zellnester die gleiche ist. Bereits makroskopisch zwang die Form des Tumors, der vollständige Mangel jeglicher papillärer Wucherungen, die beim Carcinoma penis so häufig sind, ferner der Mangel irgend welcher Ulceration, die so früh an dem Carcinom aufzutreten pflegt, von der Annahme eines solchen abzusehen. Ich habe weiter erwähnt, dass die Grösse der Zellnester den Befunden bei den häufig zur Untersuchung kommenden Cancroiden nicht entspricht. So umfangreiche Zellnester, die mehrere Millimeter Durchmesser erreichen, pflegt man bei Cancroiden nicht anzutreffen, ganz abgesehen von den fast regelmässig darin sich findenden centralen Höhlen, denen eine eigene, noch weiter zu erörternde Bedeutung zukommt. Vielleicht würde zwar im weiteren Verlauf das Bild betreffs der Grösse der Zellnester einem 
gewöhlichen Cancroid ähnlicher geworden sein, denn, wie erwähnt, zeigen viele der grossen epithelialen Nester das beginnende Eindringen von Stroma an ihrer Peripherie, und in späteren Stadien wären vielleicht an Stelle dieser grossen epithelialen Felder solche von klein alveolärem, etwas mehr krebsähnlichem Bau zu finden gewesen. In der jetzt vorhandenen Grösse und Form pflegen sie beim Cancroid jedoch nicht vorzukommen. Sodann werden anch so seltene und zugleich in so grossen Abständen von einander befindliche Verbindungen mit der Oberfläche, wie in unserem Fall, bei jenen nicht beobachtet. Bei einem Cancroid würde die Epithelwucherung an einer umschriebenen, mehr oder weniger grossen Partie der Glans begonnen und von da sich weiter ausgebreitet haben, so wie beim Drüsenkrebs ein Theil einer Drüse, eine Drüse oder eine Gruppe benachbarter Drüsen in Wucherung zu gerathen und so den Ausgangspunkt zu bilden pflegt. Hier dagegen muss ein fast gleichzeitiger Beginn an fast gleichmässig vertheilten Stellen der Oberfläche der Glans angenommen werden mit in ähnlicher Weise localisirten Nachschüben, - als welche ich die weniger tief gehenden, noch in der Albuginea endigenden Epithelzapfen, die auch ziemlich gleichmässig auf der ganzen Glans vertheilt sind, in Anspruch nehme, - eine Entstehungs- und Wachsthumsweise, die wohl auf eine besondere, von den gewöhnlichen Krebsen abweichende Natur des Tumors hindeuten dürfte: In dieser Annahme bestärken auch die weiteren Befunde.

Neben den Epithelzellnestern und meist in nächster Nähe derselben finden sich Heerde von Granulationsgewebe, die grosse Mengen von Lymphkörpern aufweisen, und deutliche Riesenzellen mit peripherischen Kernen; in manchen sind die Riesenzellen zahlreich, in anderen spärlich, in kleineren fehlend. In Bezug auf die Genese dieser Heerde scheint das wahrscheinlichste zu sein, dass entweder die grossen epithelialen Zellnester in ihrer Eigenschaft als Fremdkörper die Ursache zu ihrer Bildung sind, oder dass der Urin, welcher bei ganzem oder theilweisem Abschluss der Urethralöffung das Gewebe der Glans infiltrirte, durch seine Gegenwart chemisch oder mechanisch reizend die Granulationen hervorrief, wie ich auch den grösseren Theil der kleinzelligen Infiltration, die namentlich die nächste Umgebung der Epithel- 
zellnester in so reichlichem Grade einnimmt, auf dessen Rechnung setzen möchte. Dass auch der Reiz der Zellnester mit in Frage kommt, beweist die kleinzellige Infiltration, die sich in der Nachbarschaft der meisten carcinomatösen Neubildungen nachweisen lässt, doch wohl nicht häufig so reichlich wie hier.

Auf Gegenwart von Bakterien sind die Veränderungen nicht zurückzuführen, da die Untersuchung auf solche vermittelst der verschiedensten Färbemethoden stets negativ ausfiel. Impf-, bezw. Züchtungsversuche konnten nicht mehr angestellt werden. Es bleibt also nur die Annabme übrig, dass einerseits die epithelialen Einlagerungen, andererseits die Durchtränkung der Gewebe mit Urin den Reiz erzeugten, der sowohl Bildung von Granulationsgewebe, wie Infiltration mit Rundzellen bedingte.

Auffallend ist noch der Befund von so ungemein zahlreichen Ehrlich'schen Mastzellen und den eigenthümlichen homogenen Kugeln. Ich führe beide so unmittelbar neben, bezw. mit einander an, weil es mir scheint, als wenn sie zu den Veränderungen, welche das sie enthaltende Gewebe aufweist, oder doch zu dem Reizzustand, in welchem sich dasselbe befindet, in dem gleichen oder einem ähnlichen Verhältniss stehen. Dass die Ehrlich'schen Mastzellen in normalem Bindegewebe vorkommen, ist bekannt, doch ist darin die Zahl nur eine geringe. Eine bemerkenswerthe Vermehrung im Verlaufe von acuten Entzündungen habe ich nicht constatiren können, auch ist mir nicht bekannt, dass dieses von anderer Seite geschehen wäre. Um so auffallender ist es, dass bei dem vorliegenden Fall, wo seit 7 Jahren entzündliche Reize in wechselnder Intensität vorhanden gewesen sind, die Mastzellen in so zahlreichen Exemplaren sich constatiren lassen.

Auch die homogenen Kugeln, welche in so grosser Anzahl, meist in Gruppen und von wechselnder Grösse in dem Tumor sich finden, habe ich in Schnitten von der normalen Glans, zwai nur in wenigen Exemplaren, einzeln oder in Gruppèn von 2-3, aber doch sicher nachweisen können. Dass hier eine Vermehrung unter dem Einflusse der Entzündung stattgefunden hat, möchte ich, wie von den Mastzellen, annehmen und möchte, wie bei diesen, auf die lange Dauer ein besonderes Gewicht legen. Thr Vorkommen im normalen Gewebe schliesst wohl die Annahme aus, 
dass ihre Vermehrung zu der Entzüudung in ursächlichem Zusammenhange stehe, im Gegentheil scheinen andere Reize, welche chronisch auf die Gewebe einwirken, einen Einfluss auf ihre Vermehrung zu haben.

Es findet die Annahme einer Vermehrung von, normaler Weise in den Geweben vorhaudenen Gebilden eine Stütze in dem, was Sachs über das Vorkommen von gleichen oder doch sehr nahe verwandten in der Magenschleimhaut sagt. Wie schon erwähnt, hat Sachs im "Archiv für experimentelle Pathologie" Bd. 24, Beschreibung und Abbildung von hyalinen Kugeln, bezw. Schollen gegeben, deren Fundort sowohl die normale, wie die pathologisch veränderte Magenschleimhaut war. Auch er constatirt, dass sie im normalen Magen nur vereinzelt vorkommen, hingegen unter pathologischen Verhältnissen in sehr grosser Zahl auftreten.

Kugeln gleichen Aussehens und gleichen Verhaltens gegen Reagentien haben Cornil und Alvarez beim Rhinosclerom gefunden, nur nicht frei in Gewebslücken and Spalten, sondern gruppenweise in grossen Zellen eingeschlossen; sie beschreiben dieselben in einem Aufsatz über das Rhinosclerom in den Archives de Physiologie von Brown-Séquard und Charcot. 1885. 2 Sém. III. Sér. Tom. 6 p. 24 und 25 . Sie ziehen eine „hyaline Degeneration" des Protoplasma in Betracht unter dem directen Einfluss der Rhinosclerombacillen, fügen aber doch hinzu, dass das seltene Vorkommen der Bacillen in der nächsten Nähe, bezw. in den betroffenen Zellen and das differente Verhalten der Kugeln und der Bacillen zu Reagentien nicht viel Wahrscheinlichkeit dafür zuliesse. Es ist also auch hier wohl der chronische Reizzustand des Gewebes als das wahrscheinlichste causale Moment für das zahlreiche Auftreten der Kugeln in Auspruch zu nehmen.

Ueber die Substanz, aus welcher die Kugeln gebildet sind und die von Sachs sowohl, wie von Cornil und Alvarez als Hyalin (nach v. Recklinghausen) angesprochen wird, kann ich mich nicht weiter auslassen; die meinigen geben durch ihre Reactionen absolut keinen Anhaltspunkt für Annahme von Amyloid, doch auch keinen solchen für Hyalin, so dass ich diese Frage noch offen lassen muss.

Es bleibt mir noch übrig, die Wege zu erklären, die sowohl 
der Urin wie der Eiter gewählt haben, um aus so zahlreichen Oeffungen auf der Oberfläche der Glans zum Vorschein kommen zu können, wie dieses am Patienten constatirt ist. Eine directe Communication der Epithelzellnester mit der Schleimhaut der Urethra konnte nicht nachgewiesen werden, desgleichen auch nicht eine solche von deren Höhlen mit dem Lumen der Urethra. Vorhanden muss dieselbe gewesen sein, wenn auch nur indirect. Ob innerhalb der Glans, ob hinter derselben, ist nicht mehr zu entscheiden, doch muss der Urin Eingangspforten in das Gewebe der Glans gefunden haben; er hat dieses durchsetzt, ist bis an die epithelialen Zellnester vor- und in sie eingedrungen und durch dieselben hindurch nach aussen gelangt. Die von mir verfolgten Oeffnungen auf der Oberfläche der Glans führen sämmtlich in Höhlen, die das Innere von Epithelzellsträngen einnehmen und die zum Theil noch Eiterzellen in grösserer oder geringerer Zahl enthalten. Es muss also sowohl Urin wie Eiter auf dem Wege der Ernährungsflüssigkeit, $d . h$. durch die intercellulären Saftlücken zwischen den Epithelzellen, deren grosse Breite ich auch hervorgehoben habe, bis in das Innere der Zellnester vorgedrungen sein. Für den Urin ist dieses nicht mehr nachzuweisen, doch kann für die Eiterkörper die Richtigkeit dieser Annahme keinem Zweifel unterliegen. Denn wirklich sind am mikroskopischen Präparat unzählige Eiterkörperchen zwischen den Epithelzellen sichtbar, von denen viele, dem Wege sich anpassend, eine langgestreckte Form angenommen haben, also augenscheinlich auf der Wanderung nach dem Lumen begriffen sind.

An manchen waren sie sogar so zahlreich vorhanden, dass sehr genaue Betrachtung nöthig war, um das Epithelzellnest nicht vor den auf der Wanderung begriffenen Eiterzellen zu übersehen.

Ich kann die Resultate meiner Untersuchung in Folgendem kurz zusammenfassen:

1. Die unter dem Bilde einer Cavernitis chronica verlaufende Erkrankung der Urethra ist im Wesentlichen als eine epitheliale Neubildung eigener Art aufzufassen, indem an ziemlich gleichmässig vertheilten Partien der Oberfläche von den geschichteten Epithelien der Glans epitheliale Zellstränge ausgehen, die sich zu ausserordentlich grossen, umfangreichen Zellnestern aus- 
breiten, welche, durch schmale Ausläufer untereinander zusammenhängend, das Gewebe der Glans durchsetzen.

2. Von einem gewöhnlichen Cancroid unterscheidet sich die Neubildung durch Entstehung, Verlauf und Symptome, durch das makroskopische Verhalten, durch die Grösse und topographischen Verhältnisse der Epithelzellnester.

3. Schon frühzeitig verursachte die Neubildung eine Verengerung, später Verschluss des Orificium urethrae. Der Urin bahnte sich Wege bis in das Innere der Epithelzellnester, welche Höhlen aufweisen, und entleerte sich durch zahlreiche Fisteln nach aussen, die den Stellen entsprechen, an welchen die Epithelzellnester mit dem Oberflächenepithel zusammenhängen, und die in die Höhlen der Zellnester sich fortsetzen.

4. An Stelle des Corpus cavernosum glandis finden sich lockeres Bindegewebe und Bündel glatter Muskelfasern mit diffuser und heerdweiser Infiltration, namentlich in der Nähe der Epithelzellnester. Ferner sind Heerde von Granulationsgewebe mit mehrkernigen Riesenzellen vorhanden.

5. In dem faserigen Bindegewebe finden sich zahlreiche Mastzellen (nach Ehrlich) und zahlreiche meist gruppenweise zusammenliegende eigenthümliche homogene Kugeln.

Zum Schlusse sei es mir gestattet, Herrn Prof. Dr. Langhans für die gütige Ueberlassung des Materials und für die bereitwillige Unterstützung, die er mir bei dieser Arbeit hat zu Theil werden lassen, sowie Herrn Prof. Dr. Kocher für die Ueberlassung der Krankengeschichte meinen besten Dank auszusprechen. 